\section{Jean-François Démonet Richard Wise Richard S. J. Frackowiak}

\title{
Les fonctions linguistiques explorées en tomographie par émission de positons
}

Les progrès récents de la tomographie par émission de positons permettent actuellement une approche de l'anatomie fonctionnelle des aires du langage dans le cerveau de sujets normaux. L'interprétation des résultats est rendue difficile par divers problèmes méthodologiques concernant l'activation cérébrale soustractive, ainsi que par certains $a$ priori théoriques concernant la nature des relations cerveau/langage. De nouvelles approches méthodologiques sont décrites : l'analyse corrélationnelle des activités cérébrales régionales, qui rend compte de l'anatomie distribuée des fonctions linguistiques, et l'étude des activations chez un sujet donné, qui permet de contrôler les facteurs de variabilité inter-individuelle tant au niveau anatomique que cognitif.

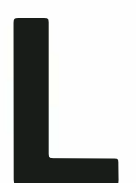

es méthodes d'exploration fonctionnelles cérébrales, développées depuis une quarantaine d'années, fournissent désormais des données complémentaires à celles de l'aphasiologie classique, qui doivent être intégrées à une conception d'ensemble de la biologie du langage. Par l'amélioration croissante de leur résolution spatiale, les techniques isotopiques d'imagerie tomographique du cerveau humain, telles que la tomographic par émission de positons, donnent accès à une anatomie fonctionnelle de nombreux systèmes neuronaux, y compris ceux qui supportent les fonctions cognitives telles que la mémoire et le langage. L'examen attentif des résultats obtenus dans ce domaine par différentes équipes montrent des discordances qui ne remettent pas en cause la validité de cette approche mais qui soulignent l'importance cruciale d'aspects méthodologiques sur lesquels est centré cet article.

\section{Rappels méthodologiques}

Les techniques isotopiques d'imagerie tomographique du cerveau consistent en le recueil de signaux photoniques émis par un isotope administré au sujet et dont les variations constituent des indices, plus ou moins directs, de l'activité cérébrale durant la période d'acquisition de ces signaux. L'isotope est, soit un émetteur de photon unique dans le cas de la technique SPECT, soit un émetteur de positon, puis d'une paire de photons, pour la technique PET (en français, tomographie par émission de 


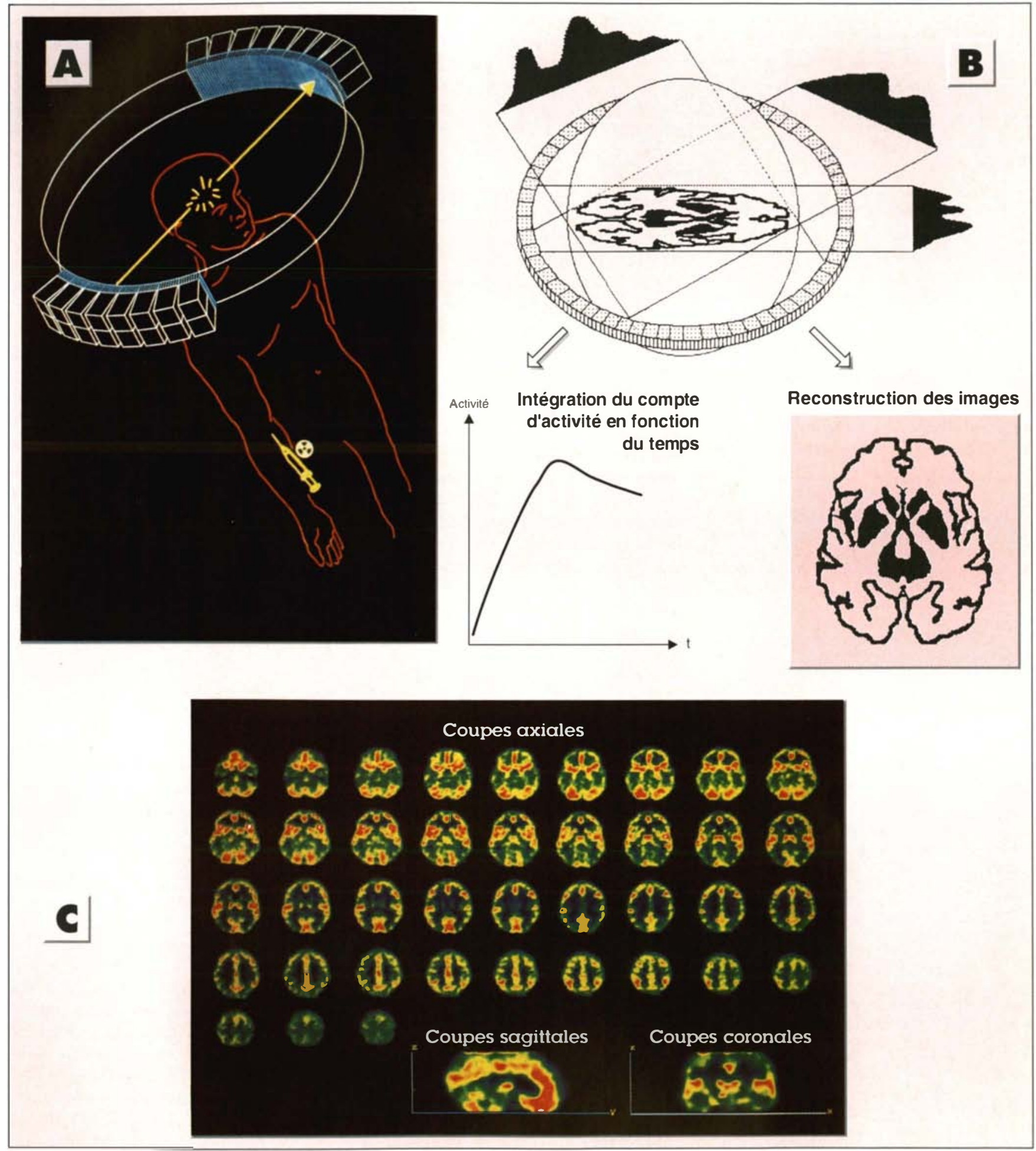

Figure 1. A. Principe du recueil du signal dans une caméra à émission de positons : une paire de photons émis à $180^{\circ}$ est recueillie au niveau de récepteurs disposés en anneau autour de la tête du sujet. B. Principe d'intégration dans le temps des signaux et de reconstruction des images du cerveau en coupe axiale. C. Série d'images axiales et reconstructions dans le plan sagittal et dans le plan coronal obtenues en effectuant la moyenne de six mesures successives pratiquées chez un sujet.

$\mathrm{m} / \mathrm{s} n^{\circ} 8-9$ vol. 9, août-septembre 93 


\section{RÉFÉRENCES}

1. Risberg J. Regional cerebral blood flow measurements by 133 Xe-inhalation : methodology and applications in neuropsychology and psychiatry. Brain Language $1980 ; 9: 9-34$

2. Petersen SE, Fox PT, Posner MI, Mintun M, Raichle ME. Positron emission tomographic studies of the cortical anatomy of single-word processing. Nature 1988 ; 331 : 585-9.

3. Posner MI, Petersen SE, Fox PT Raichle ME. Localization of cognitive operations in the human brain. Science 1988 $240: 1627-31$

4. Petersen SE, Fox PT, Posner MI, Mintun M, Raichle ME. Positron emission tomographic studies of the processing of single words. J Cognitive Neurosci 1989; 1: 153-70.

5. Petersen SE, Fox PT, Snyder AZ Raichle ME. Activation of extrastriate and frontal cortical areas by visual words and word-like stimuli. Science 1990; 249 : 1041-4.

6. Sergent J, Ohta S, MacDonald B. Functional neuroanatomy of face and object processing. Brain $1992 ; 115: 15-36$.

7. Sergent J, Zuck E, Levesque M, Mac Donald B. Positron emission tomography study of letter and object processing : empirical findings and methodological considerations. Cerebral Cortex $1992 ; 2$ : 68-80.

8. Zatorre RJ, Evans AC, Meyer E, Gjedde A. Lateralization of phonetic and pitch discrimination in speech processing. Science $1992 ; 256$ : 846-9.

9. Wise RJ, Chollet F, Hadar U, Friston K, Hoffner E, Frackowiak R. Distribution of cortical neural networks involved in word comprehension and word retrieval. Brain 1991 ; $114: 1803-17$. positons) dont la résolution spatiale et la sensibilité sont supérieures à celles de la SPECT. Les derniers progrès de la technique PET permettent actuellement d'obtenir des mesures de l'activité fonctionnelle du cerveau dans des volumes de l'ordre du $\mathrm{mm}^{3}$ et couvrant l'ensemble du cerveau. $\mathrm{La}$ résolution spatiale de ces appareils est donc tout à fait satisfaisante, du même ordre que celle des meilleures techniques d'imagerie morphologique du cerveau, comme l'imagerie par résonance magnétique nucléaire. Après l'acquisition des signaux, des programmes informatiques de reconstruction permettent l'obtention d'images, ou de coupes, au sein desquelles la valeur des pixels correspond à l'intensité du signal (ou activité) provenant de nombreux points différents du volume cérébral (figure 1). L'intégration au cours du temps (période d'acquisition des signaux) des valeurs d'activité recueillies peut permettre, lorsque l'on utilise un radio-traceur à très courte demi-vie lié à une molécule organique tel que l'oxygène 15 lié à l'eau ou au $\mathrm{CO}_{2}$, de calculer des valeurs relatives ou absolues correspondant au débit sanguin cérébral moyen au sein de petits volumes du cerveau ( $\mathrm{rCBF}$, en français débit sanguin cérébral régional). Cette méthode aboutit ainsi au niveau cérébral à une cartographie fonctionnelle précise, en termes de débits sanguins cérébraux régionaux.

La méthode d'activation, associée à ces techniques, est fondée sur la comparaison des activités observées, d'une part, lors d'une condition " de base " ou " de référence " et, d'autre part, dans une ou plusieurs conditions expérimentales "actives" au cours desquelles des variations sont appliquées à l'état physiologique du sujet. L'indice d'activité cérébrale étudié dans ce cas est le plus souvent le débit sanguin cérébral, étroitement couplé à l'activité métabolique au sein de petits volumes de tissu cérébral. La soustraction des valeurs régionales observées au cours de la condition "de référence " à celles enregistrées au cours de la condition " active" permet de définir les régions dans lesquelles des variations sensibles sont présentes d'une condition à l'autre et qui sont donc impliquées dans la (les) fonction(s) physio- logique(s) qui étai(en)t l'objet de l'expérience. Une validation générale de cette méthode soustractive a été obtenue depuis trois décennies (au départ avec des techniques plus grossières de mesure du débit sanguin) par l'obtention d'une importante augmentation du débit sanguin (ou activation cérébrale) dans les régions correspondant aux cortex primaires sensoriel ou moteur en comparant une condition de base sans stimulation et une condition "active" au cours de laquelle une stimulation sensorielle ou motrice est effectuée. Rapidement, la méthode d'activation a été étendue à des conditions expérimentales d'ordre " cognitif ", visant à l'identification, chez des sujets normaux, des corrélats cérébraux des fonctions de compréhension ou d'expression du langage ou des fonctions mnésiques. De nombreux travaux ont donc vu le jour dans ce domaine depuis plusieurs décennies, les premiers utilisant des techniques d'imagerie isotopique monophotonique à détecteurs statiques [1], et les plus récents (voir plus bas) étant suscités par les progrès de la méthodologie PET, à partir du premier travail de Petersen et al. [2].

\section{Principaux résultats}

De nombreux articles récents ont donc été consacrés aux corrélats cérébraux des fonctions linguistiques. Ils sont dus, notamment, aux équipes de Saint Louis [2-5], de Montréal [6-8] et de Londres [9-12]. Le caractère varié des méthodologies et des paradigmes utilisés ne permet pas de rendre compte ici de façon détaillée de la littérature disponible même dans le domaine particulier des activations "linguistiques ". D'une façon générale, ces études retrouvent les effets d'activation aux " entrées " et " sorties " du " système cognitif " (voir par exemple [2]) ainsi que la prépondérance de l'activation hémisphérique gauche pour les fonctions linguistiques chez les sujets droitiers. A titre d'exemple d'opposition forte entre activités cérébrales liées à des tâches linguistiques d'une part et non linguistiques d'autre part, la figure 2 (tirée d'un de nos travaux récents, [11]) présente le résultat de comparaisons effectuées, dans le même groupe de sujets normaux droitiers, 


\section{RÉFÉRENCES}

10. Howard D, Patterson K, Wise R, Brown WD, Friston K, Weiller C, Frackowiak R. The cortical localization of the lexicons : PET evidence. Brain 1992; 115 : 1769-82.

11. Démonet JF, Chollet F, Ramsay S, Cardebat D, Nespoulous JI, Wise R, Rascol A, Frackowiak RSJ. The anatomy of phonological and semantic processing in normal subjects. Brain 1992 ; 115 : 1753-68

12. Price $\mathrm{C}$, Wise $\mathrm{R}$, Ramsay $\mathrm{S}$, Friston $\mathrm{K}$, Howard D, Patterson K, Frackowiak R. Regional response differences within the human auditory cortex when listening to words. Neurosci Lelt 1992 ; 146 : 179-82.

13. Churchland PS. Neurophilosophy : Toward a Unified Understanding of the Mind/Brain. Cambridge : MIT Press, 1986.

14. Mesulam MM. Large-scale neurocognitive networks and distributed processing for attention, language and memory. Ann Neurol 1990 ; 28 : 597-613.

15. Fox PT, Raichle ME. Stimulus ratc determines regional brain blood flow in striate cortex. Ann Neurol $1985 ; 17$; 303-5.

16. Frith CD, Kriston KJ, I,iddle PF, Frackowiak RSJ. A PET study of word finding. Neuropsychologia 1991 ; 29 : 1137-48.

17. Talairach J, Tournoux P. Co-Planar Stereotaxic Atlas of the Human Brain. 3-Dimensional Proportional System: an Approach to Cerebral Imaging. Traduction Mark Rayport. New York: Thieme Medical Publishers, Inc. Stuttgart, New York : George Thieme Verlag, 1988.

18. Friston KJ, Frackowiak RSJ. Imaging functional anatomy. In : I assen NA, Ingvar DH. Raichle ME, Friberg, eds. Brain Work and Mental Activity. Copenhague : Munksgaard, 1992.

$\mathrm{m} / \mathrm{s}$ n० 8-9 vol. 9, août-septembre 93 entre deux tâches verbales (l'une phonologique et l'autre lexico-sémantique) et une tâche non verbale (détection de tons aigus dans des triplets de tons purs). Lorsque la tâche non verbale est prise comme tâche "de référence " dans la comparaison, des augmentations très significatives du débit sanguin sont observées pour les mesures effectuées au cours des tâches verbales, majoritairement situées dans des régions de l'hémisphère cérébral gauche. Lorsque la comparaison inverse est effectuée (les tâches verbales servant alors de référence), l'activation cérébrale liée à la tâche non verbale est localisée dans l'hémisphère cérébral droit. Concernant la nature plus précise des structures anatomiques impliquées spécifiquement dans les fonctions du langage, nous nous limiterons à la présentation de quelques résultats convergents disponibles dans la littérature et obtenus à l'aide de différents paradigmes expérimentaux.

L'implication de l'aire de Wernicke (moitié postérieure du gyrus temporal supérieur gauche) dans la compréhension du langage parlé a été décrite, au siècle dernier, par l'auteur allemand dont le nom est attaché à cette portion du cortex associatif auditif. La plupart des études en PET ont retrouvé une activation dans cette région associée aux tâches de traitement de stimuli linguistiques perçus auditivement $[2,4,8,9,11]$ (figures $2 A$ et $2 B$ ). Si, dans ce dernier travail, nous avons également observé une très nette prépondérance hémisphérique gauche dans l'activation de cette région temporale supérieure, cette étude montre cependant également une activation des régions homologues temporales droites (visible sur la figure 2), à un moindre degré d'intensité, ce qui évoque un rôle accessoire de ces régions droites dans la compréhension du langage, rôle qui pourrait devenir majeur pour la récupération d'une aphasie par lésion temporale gauche. Si le rôle de l'aire de Broca (pied de la $3^{e}$ circonvolution frontale gauche) dans les processus de préparation et de programmation des éléments du langage parlé est bien connu, plusieurs études très récentes viennent de montrer son implication dans des tâches pour lesquelles, en apparence, aucune production linguistique ne s'effectue : tâche de catégorisation de lettres sur la base de leur équivalence phonétique (en anglais, lettres "rimant " en $i$ comme B, ..., opposées à $H, \ldots$ ) [7], tâche de jugement de rime sur des paires de syllabes [8], tâche sérielle de détection de phonème sur des non-mots (suites de syllabes sans signification) [11] (figure $2 A)$. L'activation d'une zone prémotrice dans ces tâches perceptives de type métalinguistique (c'est-à-dire éloignées des conditions "naturelles" de l'usage du langage) suggère l'intervention d'un transcodage sensori-moteur proche des phénomènes de langage intérieur, de mémoire de travail auditivo-verbale ou des stratégies développées par l'enfant lors de l'acquisition du langage. Ce transcodage hautement routinisé et quasi latent dans des conditions habituelles de compréhension du langage pourrait être réactivé dans les conditions artificielles créées par les tâches d'activation.

La nature précise des substrats neuronaux des processus de lecture ou des processus lexicaux et sémantiques est beaucoup moins bien établie que les rôles des aires de Wernicke et de Broca. Dans deux études récentes $[10,11]$ ainsi que dans d'autres résultats préliminaires, un certain accord se fait jour cependant pour imputer à la région temporale moyenne et postérieure gauche un rôle dans les processus d'accès au lexique, alors que des zones plus bas situées dans le lobe temporal gauche, ainsi qu'à la jonction occipito-temporale gauche, pourraient être impliquées dans l'accès aux représentations sémantiques des objets $[6,11]$. Dans ce dernier travail, en utilisant une tâche de catégorisation sémantique double sur des paires adjectif-nom concret, nos résultats impliquent en fait l'intervention de zones très distribuées dans l'hémisphère gauche (figure $2 B$ ), incluant non seulement les régions temporales postéro-inférieures mais aussi la région du carrefour temporopariétal (jonction des aires 40/39 de Brodmann) ainsi que des zones préfrontales supérieures et cingulaires postérieures. Il faut souligner que Wise et al. (communication personnelle) retrouvent également l'implication de régions pré-frontales et du carrefour temporo-pariétal gauches 

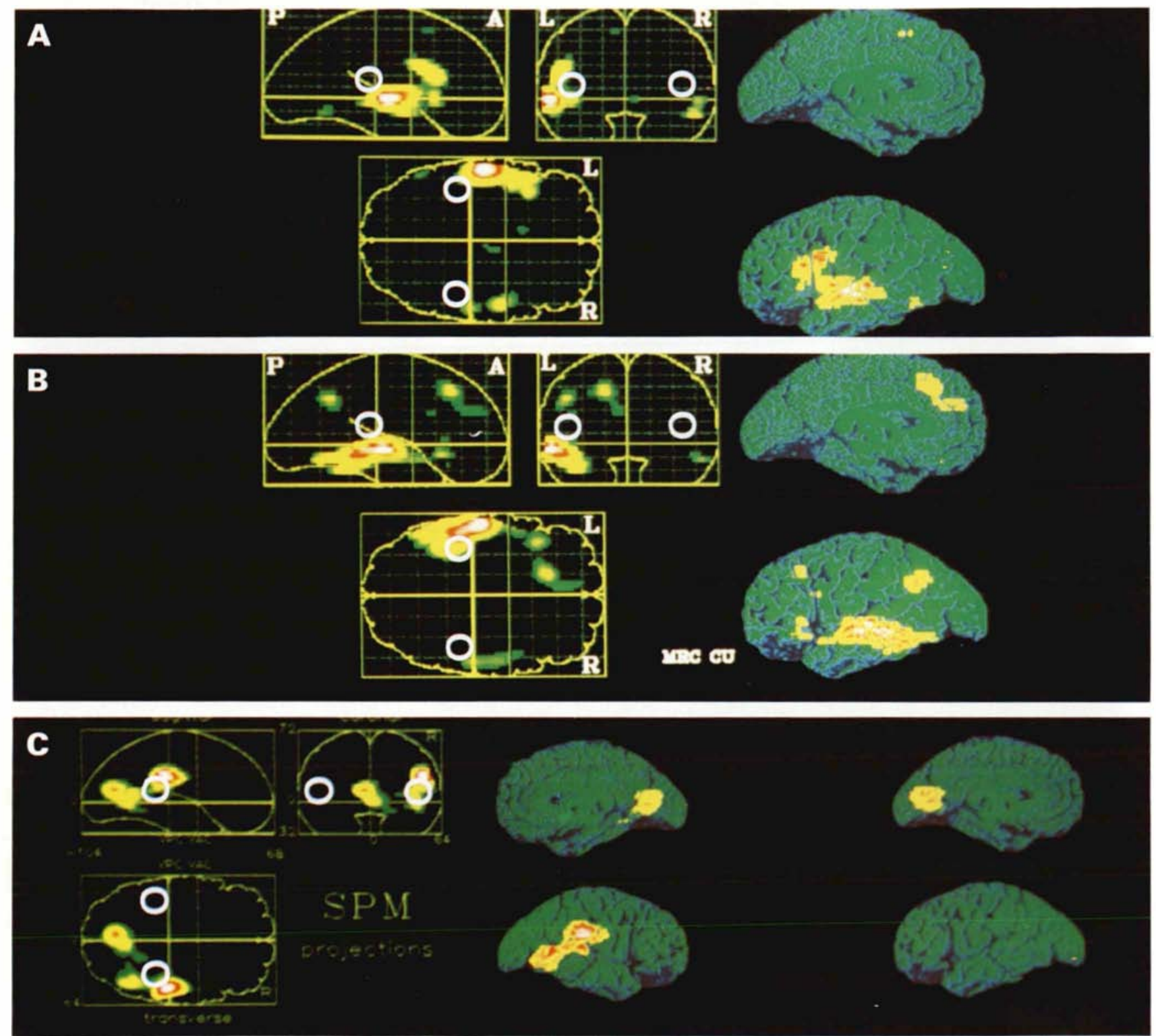

Figure 2. Trois cartes de significativité statistique (test $t, p<0,001$ ) pour des comparaisons soustractives entre deux tâches verbales et une tâche non verbale [11], montrant la projection des pixels significatifs dans I'espace tridimensionnel de Talairach et Tournoux [17], et selon la méthode statistical parametric mapping (SPM) [18]. Sont représentées trois vues en transparence du volume cérébral: vue sagittale, vue coronale (section du volume cérébral dans le plan frontal) et vue axiale (partie gauche de la figure) et des vues de la surface corticale interne et externe (partie droite de la figure) pour l'hémisphère cérébral gauche uniquement dans les parties $A$ et $B$ pour les deux hémisphères dans la partie C. A : Résultats de la comparaison soustractive : détection phonémiquedétection de tons. L'activation se situe principalement dans l'aire de Wernicke (en blanc), I'aire de Broca (en rouge), et accessoirement en temporal supérieur droit et temporal inférieur gauche (en jaune-vert). B : Résultats de la comparaison soustractive : catégorisation sémantique-détection de tons. L'activation se situe principalement dans l'aire de Wernicke (en blanc) et d'autres zones moyennes et inférieures du lobe temporal gauche (en rouge), au niveau du carrefour temporo-pariéto-occipital gauche (en rouge) et de la région préfrontale supérieure et interne gauche (en jaune); plus accessoirement, discrètes activations en frontal inférieur gauche et temporal supérieur droit (en jaune-vert). C : Résultats de la comparaison soustractive: détection de tons-détection phonémique. L'activation se situe principalement dans la région pariétale inférieure et temporale supérieure droites (en blanc-rouge). Elle s'étend également en temporal inférieur droit et cingulaire postérieur (en jaune-rouge). Dans les trois comparaisons, la région du cortex auditif primaire (droit et gauche) n'est pas activée (zones cerclées de blanc). 
lors d'une tâche de production " silencieuse" (sans expression verbale à voix haute) de verbes sémantiquement associés à des noms présentés auditivement.

\section{Discussion}

Les travaux brièvement décrits plus haut ont théoriquement une valeur heuristique certaine car ils permettent d'acquérir sur les substrats cérébraux du langage, chez des sujets normaux, des informations par des voies indépendantes du cadre conceptuel, jusque-là pratiquement unique, de la méthode anatomo-clinique confrontant chez des malades aphasiques la localisation des lésions et la nature des dysfonctionnements linguistiques observés. Par ailleurs, cette inflation d'études et de résultats prend place dans le cadre de l'essor actuel des sciences cognitives et d'un débat épistémologique autour de la pertinence du réductionnisme [13] des neurosciences à l'égard de la psychologie. Dans ce contexte théorique très général, et parfois très conflictuel, les résultats de la plupart de ces études d'activation linguistique ont reçu une interprétation localisationniste ou associationniste dont l'illustration la plus claire peut être trouvée dans Petersen et al. ([2] voir la figure 3 présentée par ces auteurs): un foyer d'activation cérébrale (par exemple, dans l'hémisphère gauche, l'aire 47 dans la nomenclature de Brodmann) est identifié à un processus psycholinguistique (par exemple, l'association sémantique). Certaines divergences dans les résultats des différentes études mentionnées plus haut, jointes aux conceptions contemporaines des substrats neuronaux des fonctions

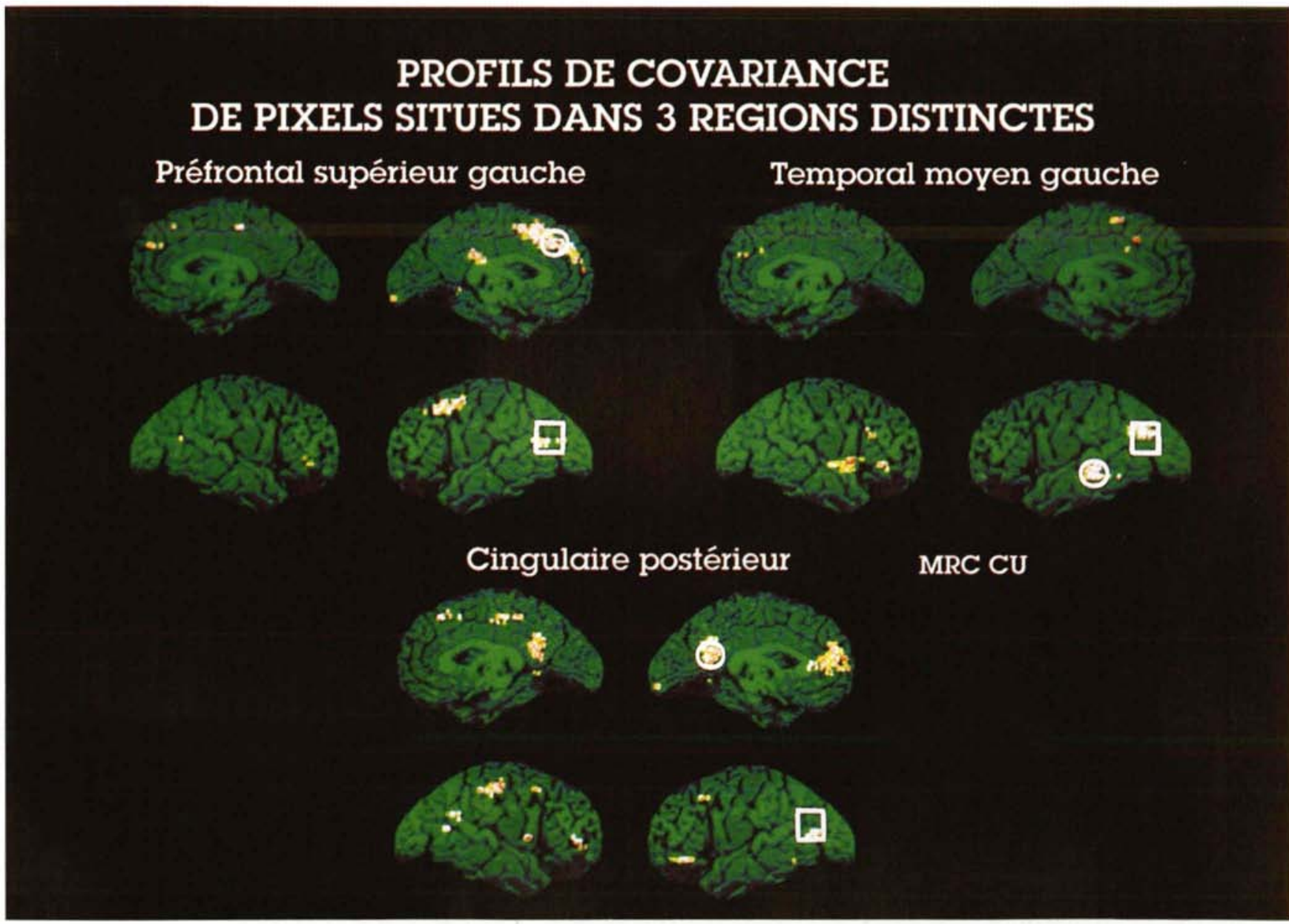

Figure 3. Trois cartes de significativité statistique $(p<0,001)$ dans l'espace stéréotaxique de Talairach et Tournoux [17] avec projections des pixels significatifs sur les surfaces corticales interne et externe des deux hémisphères cérébraux. La figure montre les profils de covariance pour trois pics d'activation (zones cerclées de blanc) observés dans une tâche lexico-sémantique [11] : région préfrontale supérieure gauche (coordonnées dans l'espace stéréotaxique $\mathrm{x}, \mathrm{y}, \mathrm{z}$ : $-16,28,44)$, région temporale moyenne gauche $(\mathrm{x}, \mathrm{y}, \mathrm{z}:-48,-36$, - 8), région cingulaire postérieure $(-2,-60,20)$. Dans chacun des trois profils, les pixels colorés en dehors du pic d'activation considéré (zone cerclée de blanc) correspondent à des régions corticales dont les variations de débit sanguin sont positivement et significativement corrélées à celles du pic d'activation. Au niveau du carrefour temporo-pariéto-occipital gauche (zone délimitée par un carré blancl, on remarque la présence de pixels covariant avec chacun de ces trois pics d'activation. Ces profils de covariance suggèrent l'existence de réseaux fonctionnels largement distribués au sein des hémisphères cérébraux.

$m / s \quad n^{\circ} 8-9$ vol. 9, août-septembre 93 
cérébrales favorisant une organisation en réseaux largement distribués au sein du cerveau [14] plutôt qu'une " mosaïque " de " centres" adjacents, conduisent à remettre en cause une vision aussi simplificatrice et à souligner le caractère crucial de certains problèmes méthodologiques pour un champ de recherche qui reste néanmoins prometteur.

Sans entrer dans des considérations techniques très délicates (relatives notamment aux problèmes de standardisation des images et de repérage des structures anatomiques), on évoquera en premier lieu certaines limitations de la technique de mesure. Si la résolution spatiale de la technique PET est actuellement satisfaisante, il n'en va pas de même pour sa résolution temporelle. En effet, les mesures précises du débit sanguin cérébral dans de très nombreuses petites régions du cerveau impliquent actuellement un temps d'acquisition de l'ordre de la minute. Or on sait, à travers l'étude des temps de réaction ou des potentiels évoqués, que la dynamique des processus cognitifs peut être de l'ordre du centième de seconde. Cette durée d'acquisition des données en PET, très longue à l'échelle des processus cognitifs, induit des contraintes majeures sur la nature des tâches cognitives utilisées. Il faut, en effet, construire des tâches dans lesquelles, durant toute la période d'acquisition, les processus cognitifs mis en jeu par le sujet étudié restent à peu près stables; en particulier, il est important de s'assurer que le sujet n'installe pas progressivement au cours de cette période une routinisation des processus cognitifs que l'on souhaite étudier. Cette routinisation peut découler, par exemple, d'une trop grande facilité de la tâche ou de la répétition des mêmes stimuli au cours de la tâche. D'une manière générale, des tâches permettant de maintenir les sujets profondément engagés, pendant toute la période de mesure, dans les processus cognitifs que l'on souhaite étudier semblent préférables à des conditions dites "passives " dans lesquelles les sujets sont supposés " seulement " percevoir les stimuli [5], ce qui revient en fait à méconnaître la nature des processus effectivement mis en jeu par les sujets. L'une des conséquences de cette contrainte méthodologique réside dans le fait que les tâches utilisées sont nécessairement éloignées des conditions naturelles de l'usage de la langue. Dans ce contexte, il est en outre souhaitable de pouvoir disposer d'indices comportementaux externes (temps de réaction, par exemple) recueillis tout au long de la mesure et permettant de s'assurer que le sujet effectue au mieux la tâche cognitive qu'on lui a proposée. Il est donc clair que l'élaboration des paradigmes expérimentaux comporte nécessairement une phase pré-expérimentale, particulièrement cruciale, au cours de laquelle les tâches que l'on projette d'utiliser sont évaluées en termes de niveau de difficulté, d'effort attentionnel et d'effets d'apprentissage, sur des sujets appariés aux futurs sujets d'expérience en ce qui concerne l'âge et le niveau culturel.

Le deuxième type de problèmes méthodologiques dans ce domaine tient au principe même d'activation cérébrale, supposant une variation régionale de débit reliée à un changement comportemental chez le sujet étudié. L'étude de l'activation repose sur la comparaison d'une (ou de plusieurs) mesure(s) effectuée(s) au cours d'une tâche "active" (par exemple une tâche linguistique), supposée modifier l'activité cérébrale, et d'une mesure de "référence ", reflétant un état "basal " d'activité cérébrale. Cette comparaison consiste généralement en une soustraction des valeurs observées à l'état de base par rapport aux valeurs recueillies dans la tâche " active". Aucun consensus n'est actuellement perceptible dans la littérature en ce qui concerne la nature de l'état de base. De nombreux auteurs ont choisi d'utiliser un état de " repos ", dans lequel les sujets restent immobiles, subissent des perceptions sensorielles minimales et ont reçu pour consigne de ne procéder à aucune activité intellectuelle particulière. Cet état est à beaucoup d'égards insatisfaisant parce que trop peu spécifié et susceptible d'induire une large variabilité inter-individuelle. Une solution alternative est d'utiliser un état de base dans lequel on sollicite le même canal sensoriel et la même réponse motrice que dans la tâche "active ", mais en évitant de déclencher le traitement cognitif auquel on s'intéresse, en jouant sur la nature des stimuli présentés ou de la consigne donnée au sujet. Plus généralement, la nature des relations unissant les différentes tâches utilisées est l'objet de controverses. Petersen et al. $[2,4,5]$ ont utilisé des paradigmes d'activation hiérarchisés dans lesquels chaque nouvelle tâche est supposée inclure la précédente plus une nouvelle composante. C'est cette composante additionnelle qui est l'objet de l'étude et l'on en isole le corrélat, en termes d'activité cérébrale, par soustraction des mesures obtenues au cours de ces deux tâches. Même si cette approche hiérarchique semble conceptuellement séduisante, elle s'avère en fait inadéquate tant du point de vue théorique qu'en raison du fait que les résultats décrits par ces auteurs n'ont pas tous été reproduits par d'autres équipes [7, 9, 11]. Les résultats présentés plus haut dans la figure 2 peuvent illustrer cette inadéquation de l'approche hiérarchique. Dans l'étude mentionnée [11], nous avons utilisé trois tâches d'activation, deux tâches linguistiques et une tâche non verbale de détection de tons. Ces trois tâches présentaient de nombreux points communs : mêmes canaux d'entrée (auditive) et de sortie (motrice), même rythme et intensité de stimulation, aspects séquentiels des stimuli et de la tâche, etc. Si l'on considère la tâche non verbale de détection de tons comme hiérarchiquement inférieure et totalement incluse dans les deux tâches verbales, on est amené à effectuer les comparaisons soustractives entre tâches seulement dans un " sens ", c'est-à-dire en considérant la tâche de détection de tons comme "référence". L'absence d'activation au niveau du cortex auditif primaire (figure 2) est interprétée comme le reflet d'une activation "d'entrée " commune aux tâches verbales et à la tâche de détection de tons, qui n'apparaît donc pas dans les comparaisons. Cependant, les comparaisons inverses (les tâches verbales étant considérées comme "référence ") démontrent que l'effet de la tâche de détection de tons ne se limite pas au seul cortex auditif primaire: certaines régions temporopariétales droites présentent des valeurs de débit plus élevées dans la 
tâche de détection de tons que dans les tâches verbales, et ce résultat est cohérent avec des données issues de la pathologie montrant des altérations de mémoire pour des séquences de tons en cas de lésion de ces régions hémisphériques droites. Ainsi, dans cet exemple, une tâche dite de référence peut avoir des effets d'activation propres qui conduisent à rejeter l'hypothèse d'une stricte hiérarchic entre les tâches. De la même façon, lorsque Petersen et al. [2] ont exploré l'activation reliée aux processus sémantiques, ils ont choisi comme tâche de référence la répétition de mots, en supposant que les sujets l'effectueraient sans avoir recours à un traitement sémantique de ces mots et que ce traitement n'interviendrait que dans une tâche " active ", hiérarchiquement "supérieure " (production de mots reliés sémantiquement à des mots perçus auditivement). D'un point de vue théorique, la répétition de mots induit en réalité, très vraisemblablement, un accès automatique aux représentations sémantiques et est donc susceptible d'activer certaines structures cérébrales impliquées dans ce type de traitement telles que les régions temporales inféro-postérieures [11]. L'effet de ces processus automatiques permet probablement de rendre compte du fait que Petersen et al. [2] n'aient pas observé de variation majeure dans les régions temporales (activées à la fois dans la tâche de référence et dans la tâche " active "), contrairement à Wise et al. [9], utilisant une tâche proche de celle choisie par Petersen et al. [2] mais prenant pour référence une condition " de repos ". En fait, les résultats de ces derniers auteurs suggèrent que l'utilisation d'un modèle hiérarchique simple pour décrire les relations entre les différentes tâches linguistiques permet de rendre compte de façon adéquate des effets d'activation "périphérique ", liés à la présence de la stimulation des "entrées " sensorielles (auditives ou visuclles) ou de la "sortie" motrice. Dans le cas de niveaux de codage ou de représentation linguistique plus élaborés (niveaux phonologique, lexical, sémantique), les relations inter-tâches sont plus complexes qu'une hiérarchie uni-dimensionnelle. Elles doivent être envisagées comme des variations gra$\mathrm{m} / \mathrm{s} n^{\circ} 8.9 \mathrm{vol}$. 9, août-septembre 93 duées parmi ces différents niveaux de représentation dont les poids respectifs peuvent être plus ou moins forts dans une tâche donnée. Ces variations, dépendantes de la nature de la tâche, sont liées vraisemblablement à la charge attentionnelle allouée aux processus actifs à chacun de ces niveaux de représentation et prenant, sclon les cas, un caractère plus ou moins "automatique " ou plus ou moins "contrôlé ". Notre étude récente [11] déjà mentionnéc plus haut avait pour but l'étude des activations observées respectivement dans deux tâches linguistiques: une tâche de détection séquentielle de phonèmes sur des non-mots et une double tâche de catégorisation sémantique sur des mots réels. Bien que les processus phonologiques aient été évidemment présents dans les deux cas (un décodage phonologique est nécessaire au traitement de mots réels comme de non-mots), la tâche de détection de phonèmes induisait explicitement un effort particulier sur les processus phonologiques, alors que l'autre tâche faisait porter l'effort sur le traitement sémantique (et non phonologique) des mots. Les contrastes les plus importants observés dans les comparaisons inter-tâches permettaient de différencier clairement les structures impliquées respectivement dans les processus phonologiques et lexicosémantiques (figure 2).

Cependant, ces résultats suggèrent également que certaines structures impliquées dans le traitement lexicosémantique (région temporale inférieure gauche) sont aussi activées, à un moindre degré, dans la tâche phonémique et, inversement, une activation limitée a été observée pour la tâche lexico-sémantique dans le cortex frontal inférieur gauche qui était activé massivement par la tâche phonémique. Ainsi, il semble que les différences entre tâches linguistiques tiennent beaucoup moins à des contrastes qualitatifs (présence/absence de tel processus cognitif dans telle tâche) qu'à des nuances dans le niveau des ressources attentionnelles allouées respectivement aux différents processus entrant en jeu dans différentes tâches. Par ailleurs, de très importantes différences en termes d'activation cérébrale peuvent être dues à l'intervention de nombreux facteurs " secondai- res ", facilement négligés par une arithmétique simpliste de tâches hiérarchisées. Parmi ces facteurs, on mentionnera l'influence de l'intensité et de la fréquence de présentation des stimuli qui a été mise en évidence pour des stimulations visuelles simples [15] et qui vient d'être récemment démontrée pour des stimulations auditives constituées de mots [12].

Enfin, l'interprétation des résultats d'" activation " ne devrait pas conduire à négliger les phénomènes inverses de diminution du débit sanguin cérébral qui peuvent être observés, de façon concomittante à l'augmentation du débit sanguin dans certaines régions, dans d'autres régions cérébrales. Il est évident que la soustraction entre deux tâches peut être pratiquée dans le sens habituel (tâche " active " - tâche " de base ") et dans le sens inverse, la tâche de "base " étant considérée comme " active " et vice versa. Les foyers d'activation éventuellement observés dans le premier cas deviennent alors des foyers de diminution de débit. Si ce phénomène peut sembler être un simple artifice de calcul, on a vu plus haut que, lorsqu'il s'avérait cohérent avec d'autres données physiologiques, il pouvait conduire en fait à mettre en évidence d'authentiques effets d'activation liés spécifiquement aux effets de la tâche " de base " comme l'activation temporo-pariétale droite dans la tâche de détection de tons. Cependant, il reste que de véritables diminutions de débit dans certaines régions ont parfois été démontrées, par rapport à une condition "de repos ", à l'occasion d'une tâche particulière qui induisait par ailleurs une élévation du débit dans d'autres régions; l'interprétation de telles baisses de débit $[10,16]$, reste très délicate, faisant intervenir la notion d'inhibition de certaines portions de vastes réseaux fonctionnels, de rétroaction entre différents systèmes, ou d'interaction entre un système attentionnel "diffus " et des systèmes spécifiques.

\section{Perspectives et conclusions}

L'approche soustractive des phénomènes d'activation cérébrale connaissant des limites, il faut souligner l'intérêt 
d'une autre méthode d'analyse des résultats d'activation, qui consiste en l'étude des cartes de covariance des foyers d'activation, en identifiant les pixels présentant des variations corrélées avec celles observées au niveau d'un foyer d'activation. Cette méthode met en évidence, par des corrélations positives, les phénomènes de coopération fonctionnelle entre plusieurs structures anatomiquement reliées; elle s'avère donc particulièrement pertinente pour l'exploration d'un système comme le cerveau, structuré en multiples réseaux fonctionnels. Combinée à la méthode de soustraction des tâches, cette approche peut aussi permettre d'interpréter en termes d'inhibition (correspondant à des corrélations négatives) les phénomènes, assez fréquents, de baisse d'activité constatés dans certaines régions cérébrales au cours de tâches d'activation.

A titre d'exemple, la figure 3 montre les cartes de corrélation positive des pixels correspondant au pic d'activité au sein de trois foyers d'activation observés dans la tâche sémantique décrite plus haut [11]: foyers préfrontal supérieur gauche, temporal moyen gauche et cingulaire postérieur. On peut voir que, pour chacun de ces trois pics d'activation, il existe, au niveau du carrefour pariéto-temporo-occipital, des pixels covariant avec lui, dont certains seulement (a) sont communs aux trois pics et (b) appartiennent au foyer d'activation à la jonction des aires 40 et 39 de Brodmann que nous avons décrit par ailleurs dans cette tâche (voir figure $2 B$ ). Ces résultats suggèrent l'intervention de plusieurs soussystèmes fonctionnels intervenant dans la tâche proposée, certains éléments de ces sous-systèmes étant soit topographiquement proches, soit connectés entre eux au sein d'une petite région, voire communs. Compte tenu de nos connaissances sur la connectivité et la cyto-architectonie du néocortex, ce type d'organisation fonctionnelle est hautement vraisemblable [14] et pourrait représenter certains aspects des substrats de la mémoire sémantique.

L'ensemble des données rapportées ici résulte d'études de petits groupes de sujets normaux. De nouvelles avancées techniques permettent désormais d'observer l'activation linguistique chez des sujets étudiés individuellement. Ces études chez l'individu permettront une meilleure définition anatomique des foyers d'activation en se référant aux particularités anatomiques individuelles étudiées en IRM. En outre, on pourra ainsi prendre en compte les facteurs de variation inter-individuclle, notamment dans la nature et l'intensité des stratégies mises en place pour effectuer une tâche, ainsi que les conditions particulières créées par la présence d'une lésion cérébrale chez des patients présentant une aphasie. En effet, après une nécessaire étape portant sur des sujets volontaires normaux, l'approche des mécanismes de récupération ou de compensation des déficits aphasiques constitue bien l'objectif ultime de ce type de recherche

\section{Summary}

Functional neuroanatomy of language explored with positron emission tomography

Recent advances in positron emission tomography provide a way of studying the functional anatomy of language processes in the normal brain. A large amount of data has already been acquired using the subtractive activation method which consists of comparing regional metabolic brain activities between two experimental conditions, a reference condition and an active condition (including a language task). The interpretation of such results may be obscured by various methodological problems as well as conflicting theoretical issues on the nature of brain-language relationships. The exact nature of the language task and stimuli used in the activation paradigms appear to be critical for this topic. Moreover, the strictly hierarchical subtractive method (previously described by Petersen et al, [2].) is not adequate to generate appropriate contrasts between activation conditions in most language paradigms. Further progress are expected from the inter-regional correlational analysis of cerebral activities which could account for the distributed anatomy of language functions, as well as from single subject studies in which issues on anatomical and cognitive inter-individual variability may be addressed. 